\title{
Bronchoscopy Navigation beyond Electromagnetic Tracking Systems: A Novel Bronchoscope Tracking Prototype
}

\author{
Xiongbiao Luo ${ }^{1}$, Takayuki Kitasaka ${ }^{2}$, and Kensaku Mori ${ }^{3,1}$ \\ 1 Graduate School of Information Science, Nagoya University, Japan \\ 2 Faculty of Information Science, Aichi Institute of Technology, Japan \\ 3 Information and Communications Headquarters, Nagoya University, Japan
}

\begin{abstract}
A novel bronchoscope tracking prototype was designed and validated for bronchoscopic navigation. We construct a novel mouth- or nose-piece bronchoscope model to directly measure the movement information of a bronchoscope outside of a patient's body. Fusing the measured movement information based on sequential Monte Carlo (SMC) sampler, we exploit accurate and robust intra-operative alignment between the pre- and intra-operative image data for augmenting surgical bronchoscopy. We validate our new prototype on phantom datasets. The experimental results demonstrate that our proposed prototype is a promising approach to navigate a bronchoscope beyond EMT systems.
\end{abstract}

\section{Introduction}

To develop bronchoscopic navigation, accurate and stable alignment between a pre-built 3D anatomical airway model and real-time $2 \mathrm{D}$ bronchoscopic video frames remains challenging. Although EMT-based methods provide global synchronization between patients and their CT coordinate systems [12], such techniques are still constrained due to their disadvantages such as sensitivity to localization problems caused by patient movement and inaccurate measurements resulted from magnetic field distortion. Image-based schemes usually register the world and CT coordinate systems by calculating the similarities between real bronchoscopic video frames and virtual bronchoscopic images generated from CT-derived virtual bronchoscopy [34]. Since image-based techniques depend on local texture information such as bifurcations or folds, they usually provide accurate local registration between patients and their CT coordinate systems. However, such approaches fail easily to track the global trajectory of a bronchoscope due to uncertainties (e.g., bubbles) that commonly occur in bronchoscopy.

This work explores a novel bronchoscope prototype that deals with the difficulties of EMT- and image-based methods during bronchoscopic navigation. Our main contributions are summarized as follows. First, a novel bronchoscope tracking prototype was constructed and evaluated on a bronchial phantom. We originally proposed a novel external tracking method on the basis of an optical mouse (OM) sensor and demonstrated its effective and promising performance

G. Fichtinger, A. Martel, and T. Peters (Eds.): MICCAI 2011, Part I, LNCS 6891, pp. 194202, 2011.

(C) Springer-Verlag Berlin Heidelberg 2011 
for bronchoscope motion estimation. Compared to our previous work [5], this study shows the complete idea of our tracking prototype and more attractive tracking performance by combining SMC methods. Next, currently, although only $2 \mathrm{D}$ motion information of a bronchoscope can be measured by an OM sensor in our proposed prototype, such an OM sensor-based bronchoscope mockup still has several advantages in contrast to other external tracking methods, such as EMT-based. These advantages include: (1) constructing such a bronchoscope tracking model is very cheap and simple; (2) since we design the OM sensor and the rotary encoder to be located outside a patient's airway tree, such a mockup does not occupy the space of a bronchoscope tip or its working channel but the EMT system currently requires an EMT sensor to be attached at either the surface or inside the working channel of the bronchoscope tip; this constrains the movement of the bronchoscope in big bronchial branches; and (3) the OM sensor and encoder measurements are unaffected by ferromagnetic metals or conductive material within the bronchoscope; they usually distort the magnetic fields of EMT systems and cause inaccurate measurements of EMT sensors. Third, sometimes image-based approaches unavoidably misalign patients and their CT coordinates because of inherent global uncertainties, e.g., image artifacts. After a failure to register real and virtual images for several frames, an image-based algorithm usually cannot automatically recover the tracking procedure by itself due to shortages of the global insertion depth and rotation information around the running direction of the bronchoscope. We solve such a problem using an OM sensor to directly measure such global motion information of the bronchoscope and reduce opportunities to get trapped in local minima during bronchoscope tracking. Fourth, to incorporate OM sensor output for bronchoscope tracking, we introduce SMC sampler [6], which proved to be an effective means to combine different tracking sources since it can somewhat tackle situations where ambiguities occur in bronchoscopic videos [7]. Finally, we also believe our novel bronchoscope model can be conveniently and easily integrated into intra-bronchoscopy inside operating rooms without any overloads since we can attach a rotary encoder on the angle lever surface and design to fix an OM sensor on a mouth- or nose-piece that is also indispensable for a conventional bronchoscope.

\section{Novel Bronchoscope Tracking Prototype}

\subsection{Prototype Overview}

The movements of a bronchoscope are usually comprised of three parts: (1) moving it inside or outside airway trees along the running direction, (2) rotating it around the running direction, and (3) bending its bendable section by its angle lever fixed on its control head. Three parameters corresponding to three motion components are introduced to characterize the bronchoscope movements: the insertion depth inside the airway trees, rotational angle around the running direction, and the bending angle of the bendable section.

Based on these movement properties of a bronchoscope, we designed our prototype with three key functions that are clarified as follows: (1) it directly measures 


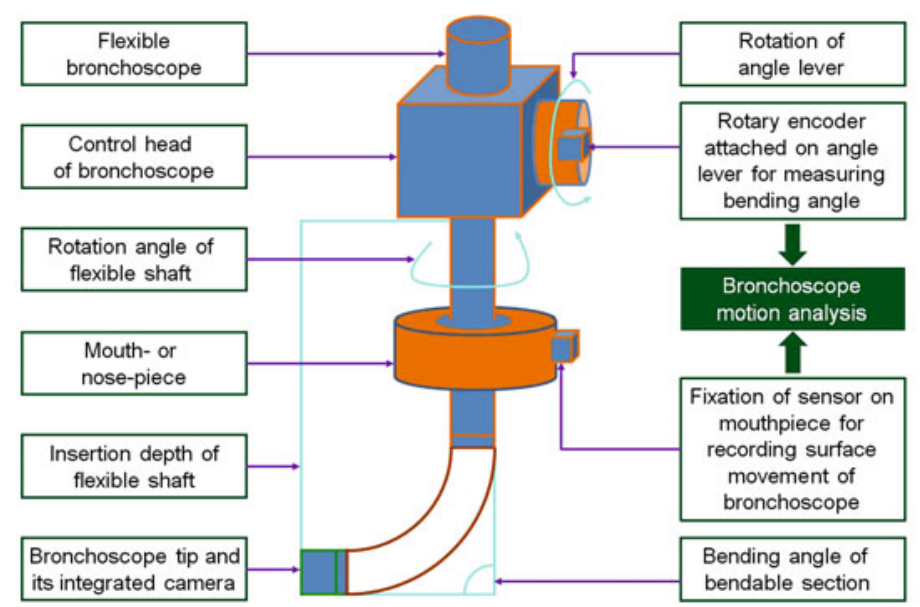

Fig. 1. Our designed bronchoscope prototype with a sensor fixed on a mouth- or nosepiece to measure insertion depth and rotation information along the running direction of a bronchoscope and a rotary encoder attached at angel lever surface to determine the bending angle of the bendable section during interventions

the insertion depth of the bronchoscope shaft, (2) it immediately records the rotational angle around the viewing (running) direction of the bronchoscope camera, (3) it automatically obtains the rotational information of the angle lever to determine the bending angle of the bendable section. Note that this movement information is acquired outside of a patient's body, and no additional sensors are attached at the bronchoscope shaft surface or its working channel. Fig. 1 outlines the prototype with different components relative to different functions.

\subsection{Bronchoscope Motion Analysis}

\section{A. Pre-processing}

Since unavoidable time-delay occurs between 2D OM sensor measurements (sample rate: $2000 \sim 6400 \mathrm{fps}$ ) and bronchoscopic images (frame rate: $30 \mathrm{fps}$ ) during data collection, we must synchronize the OM sensor outputs and video frames based on their timestamps. If the timestamps represent the exact time of two kinds of outputs, we can calibrate them through a linear interpolation.

\section{B. Fusion of OM Sensor Measurements}

After temporal calibration between the OM sensor outputs and the bronchoscopic frames, we obtain relative insertion depth $\Delta$ and rotational angle $\Psi$ of the running direction of the bronchoscope between successive bronchoscopic images. To predict six DOF parameters of bronchoscope motions, we incorporate measurements $\Delta$ and $\Psi$ into the SMC simulation because it proved to be an effective means to fuse different external tracking sources. 
Sequential Monte Carlo samples were deterministically drifted and stochastically diffused to predict the posterior probability density of interest. We here utilize an SMC simulation approach that originated from the work in 6 .

We produce set of random samples $\mathcal{S}_{i}^{k}=\left\{\left(\mathbf{x}_{i}^{k}, w_{i}^{k}, c_{i}^{k}\right): i=1,2,3, \ldots, N ; k=\right.$ $1,2,3, \ldots, M\}$ ( $N$ and $M$ are the number of frames and samples, respectively, $M$ is set to 500 since it met with a compromise between accuracy and computational complexity in our case; $w_{i}^{k}$ is a sample weight; $c_{i}^{k}$ denotes the accumulative weight of each sample) to approximate the posterior probabilistic distribution of current bronchoscope camera motion parameters $\mathbf{x}_{i}^{k}$, which are defined on the basis of transformation matrix ${ }^{C T} \mathbf{T}_{C}^{(i)}$ including translation ${ }^{C T} \mathbf{t}_{C}^{(i)}$ and rotation ${ }^{C T} \mathbf{R}_{C}^{(i)}$ from the bronchoscope camera coordinate system to the CT coordinate system at time $i$. Then our proposed bronchoscope tracking method using SMC simulation is performed in the following two steps:

State Transmission. The new state of each sample can be determined by deterministic drift and stochastic diffusion by transform function $F$ :

$$
\mathbf{x}_{i}^{k}=F\left(A \mathbf{x}_{i-1}^{k}, B n_{i}^{k}\right),
$$

where $A$ denotes a deterministic inter-frame motion and depends on the OM sensor measurements (or observations) of relative insertion depth $\Delta$ and rotational angle $\Psi$ of the running direction, $B$ describes an uncertainty or a stochastic part of the relative motion in Eq. 1, and $n_{i}^{k}$ is an independent stochastic variable.

During this state dynamic step, SMC simulation requires a probabilistic density function to present state transmission probability $p\left(\mathbf{x}_{i}^{k} \mid \mathbf{x}_{i-1}^{k}\right)$ between consecutive time steps. Since we have no prior knowledge of the bronchoscope camera movement, in other words, since we do not know prior probabilistic distribution $p\left(\mathbf{x}_{i}\right)$ for state vector $\mathbf{x}_{i}$, we employ a random walk on the basis of normal distribution with respective to noise $n_{i}^{k}: n_{i}^{k} \sim \mathcal{N}\left(\mu, \sigma^{2}\right)$ to approach dynamic density $p\left(\mathbf{x}_{i}^{k} \mid \mathbf{x}_{i-1}^{k}\right)[6]$ :

$$
p\left(\mathbf{x}_{i}^{k} \mid \mathbf{x}_{i-1}^{k}\right) \propto \frac{1}{\sqrt{2 \pi \sigma}} \exp \left(-\left(B^{-1}\left(\mathbf{x}_{i}^{k}-A \mathbf{x}_{i-1}^{k}\right)-\mu\right)^{2} / 2 \sigma^{2}\right) .
$$

Note that $A$ and $B n_{i}^{k}$ need to be determined in Eq. 11. For $A$, according to relative insertion depth $\Delta$ and rotational angle $\Psi$, we compute the relative motion with the translation vector $\Delta \hat{\mathbf{t}}_{k}^{(i)}$ and the Euler rotation angles $\Delta \hat{\alpha}_{k}^{(i)}$ as:

$$
\Delta \hat{\mathbf{t}}_{k}^{(i)}=[0,0, \Delta]^{T}, \quad \Delta \hat{\alpha}_{k}^{(i)}=[0,0, \Psi] .
$$

By transforming the rotation part from Euler angles to matrix, the deterministic drift part $A$ can be determined by

$$
A=\left(\begin{array}{cc}
\Delta^{C T} \mathbf{R}_{C}^{(i)}\left(\Delta \hat{\alpha}_{k}^{(i)}\right) & \Delta \hat{\mathbf{t}}_{k}^{(i)} \\
\mathbf{0}^{T} & 1
\end{array}\right) .
$$


Additionally, the stochastic diffusion component $B n_{i}^{k}$ can be computed by Eq. 4 in terms of $\Delta \tilde{\mathbf{t}}_{k}^{(i)}=\left[n_{t}^{k}, n_{i}^{k} t, n_{i}^{k} t\right]^{T}$ and $\Delta \tilde{\alpha}_{k}^{(i)}=\left[n_{i}^{k} \theta, n_{i}^{k} \theta, n_{i}^{k} \theta\right]$, where $t$ and $\theta$ are translational and rotational constants during stochastic diffusion.

Observation Density. After state evolution, we must compute sample weight $w_{i}^{k}$ and observation density $p\left(\mathbf{y}_{i} \mid \mathbf{x}_{i}\right)\left(\mathbf{y}_{i}\right.$ is an observation variable). In this work, $w_{i}^{k}$ is defined as the modified mean squared error (MoMSE) similarities between real bronchoscopic frame $\mathbf{I}_{R}^{(i)}$ and virtual image $\mathbf{I}_{V}$ produced from estimate $\mathbf{x}_{i}^{k}$ using volume rendering techniques; it can be presented by [4]:

$$
w_{i}^{k}=\operatorname{MoMSE}\left(\mathbf{I}_{R}^{(i)}, \mathbf{I}_{V}\left(\mathbf{x}_{i}^{k}\right)\right) .
$$

Based on factored sampling scheme [6], observation density $p\left(\mathbf{y}_{i} \mid \mathbf{x}_{i}\right)$ can be calculated by

$$
p\left(\mathbf{y}_{i} \mid \mathbf{x}_{i}=\mathbf{x}_{i}^{k}\right)=w_{i}^{k}\left(\sum_{j=1}^{M} w_{i}^{j}\right)^{-1} .
$$

Finally, the pose parameters of the current bronchoscope and its combined camera pose can be determined in terms of sample weight $w_{i}^{k}$ :

$$
\tilde{\mathbf{x}}_{i}=\max _{\tilde{w}_{i}^{k}}\left\{\left(\mathbf{x}_{i}^{k}, w_{i}^{k}\right)\right\}
$$

The final estimate corresponds to motion state $\tilde{\mathbf{x}}_{i}$ with weight $\tilde{w}_{i}$ to maximize the similarity between the current real and virtual frames.

\section{Experimental Results}

Since we currently have no patient data for our new bronchoscope tracking device, we validate the proposed tracking prototype on a phantom. We investigate three tracking schemes: (1) Deguchi et al. 4], only image registration using similarity measure MoMSE, (2) Schwarz et al. [1, using an EMT system, and (3) our new method presented in Section 2. To evaluate the tracking accuracy of the three methods, we generate two sets of ground truth data (GTD) using manual registration to align the $\mathrm{RB}$ and virtual bronchoscopic (VB) viewing points by hand. We then calculated the position and orientation errors by $\delta=\left\|\mathbf{t}-\mathbf{t}_{G}\right\|$, $\phi=\arccos \left(\left(\operatorname{trace}\left(\mathbf{R} \mathbf{R}_{G}^{T}\right)-1\right) / 2\right)$, where $\delta$ and $\phi$ are Euclidean distance and rotation error around the invariant Euler axis in accordance with estimated pose $(\mathbf{R} \mid \mathbf{t})$ and reference (ground truth) pose $\left(\mathbf{R}_{G} \mid \mathbf{t}_{G}\right)$ [9], where $\mathbf{t}$ and $\mathbf{t}_{G}$ denote translation, $\mathbf{R}$ and $\mathbf{R}_{G}$ are rotation matrices.

Table 1 summarizes the position and orientation errors by contrasting GTDs with the tracking results from the three approaches. The average position errors of the three methods were $25.5 \mathrm{~mm}, 5.09 \mathrm{~mm}$ and $1.10 \mathrm{~mm}$. Simultaneously, the average orientation error was $3.88^{\circ}$ by the proposed method, compared to $37.0^{\circ}$ and $11.1^{\circ}$ from the other two approaches. Fig. 2 illustrate the tracking accuracy of the predicted results of Experiments $B$ in contrast to the ground 
Table 1. Examples of position and orientation errors in tracking results using methods of Deguchi et al. 4], Schwarz et al. 1], and our new method

\begin{tabular}{|c|c|c|c|c|c|c|c|c|c|c|c|}
\hline \multirow{4}{*}{$\begin{array}{l}\text { GTD } \\
\text { tests }\end{array}$} & \multicolumn{11}{|c|}{ 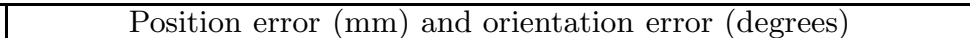 } \\
\hline & \multicolumn{3}{|c|}{ Deguchi et al. [4] } & \multicolumn{4}{|c|}{ Schwarz et al. [1] } & \multicolumn{4}{|c|}{ Our new method } \\
\hline & Position & \multicolumn{2}{|c|}{ Orientation } & \multicolumn{2}{|c|}{ Position } & \multicolumn{2}{|c|}{ Orientation } & \multicolumn{2}{|c|}{ Position } & \multicolumn{2}{|c|}{ Orientation } \\
\hline & \begin{tabular}{|l|l|} 
Mean & Std \\
\end{tabular} & Mean & Std & Mean & Std & Mean & Std & Mean & Std & Mean & Std \\
\hline $\bar{A}$ & 32.8 & $\overline{42.9}$ & 50.5 & 5.70 & 2.77 & 12.2 & 3.53 & 1.40 & 3.1 & 5.20 & 11. \\
\hline $\mathrm{B}$ & 18.2 & 31.0 & 47.1 & 4.48 & 2.66 & 10.0 & 3.38 & 0.80 & 0.50 & 1.68 & 1.37 \\
\hline ive & 25.5 & 37.0 & $\overline{48.8}$ & 5.09 & 2.72 & 11.1 & $\overline{3.50}$ & 1.10 & 1.8 & 3.88 & $\overline{6.20}$ \\
\hline
\end{tabular}

Table 2. Comparison of registered results based on visual inspection

\begin{tabular}{c||c|c|c|c}
\hline Phantom & Number & \multicolumn{3}{|c}{ Number (\%) of successfully processed frames } \\
\cline { 3 - 5 } Experiments & of frames & Deguchi et al. 4 & Schwarz et al. [1] & Our new method \\
\hline \hline $\mathrm{A}$ & 1556 & $763(49.0 \%)$ & $708(45.5 \%)$ & $1387(89.1 \%)$ \\
\hline $\mathrm{B}$ & 1498 & $832(55.5 \%)$ & $1001(66.8 \%)$ & $1462(97.6 \%)$ \\
\hline $\mathrm{C}$ & 1183 & $83(7.02 \%)$ & $948(80.1 \%)$ & $966(81.7 \%)$ \\
\hline $\mathrm{D}$ & 1805 & $198(11.0 \%)$ & $1310(72.6 \%)$ & $1582(87.7 \%)$ \\
\hline $\mathrm{E}$ & 1032 & $416(40.3 \%)$ & $538(52.1 \%)$ & $639(61.9 \%)$ \\
\hline $\mathrm{F}$ & 1228 & $606(49.5 \%)$ & $689(56.1 \%)$ & $1106(90.1 \%)$ \\
\hline \hline Total & $\mathbf{8 3 0 2}$ & $\mathbf{2 8 9 8} \mathbf{( 3 4 . 9 \% )}$ & $\mathbf{5 1 9 4} \mathbf{( 6 2 . 6 \% )}$ & $\mathbf{7 1 4 2}(\mathbf{8 6 . 0 \% )}$ \\
\hline
\end{tabular}

truth. Our new tracking prototype is more accurate and stable than the other two. Moreover, according to visual inspection, successfully processed RB frames were quantified in Table 2. Our method successfully processed a total of 7142 (86.0\%). Fig. 3 displays the RB and VB images at the selected frames by all three methods. Both prove the better accuracy and robustness of our model.

\section{Discussion}

The contributions of this work were already clarified in Section 1, Our method provides the insertion depth as global information to calculate the image similarity and hence improve its robustness at the branching cases that will be further addressed in the future. Compared to EMT-based approaches, our method will never be involved in any inherent calibration or registration such as camerasensor calibration and CT-to-physical space registration that inevitably introduce many errors before tracking with the bronchoscope. Even a bronchoscope itself contains conductive metals that distort the magnetic field and deteriorate EMT accuracy; our prototype remains free of such a problem. In contrast to image-based algorithms, our proposed method directly provides global information on the insertion depth and rotational angle of the viewing direction to predict bronchoscope motions combined with SMC simulation without any optimization procedures that get easily trapped in local minima due to the shortages of global information and such image artifacts as bubbles or patient movements. 

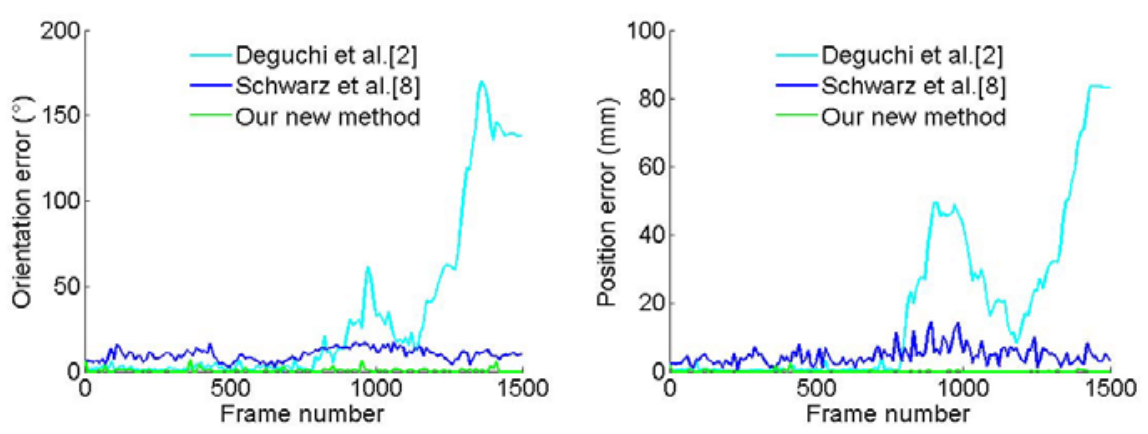

Fig. 2. Orientation error (left) and position error (right) of Experiment B are plotted from tracking results predicted by methods of Deguchi et al. 4] (cyan), Schwarz, et al. 1] (blue), and our new method (green).

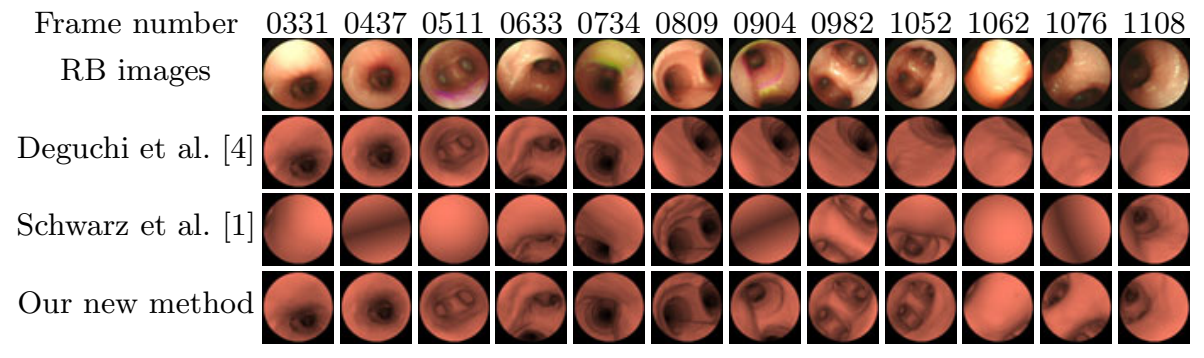

Fig. 3. Visual comparison of tracking results of Experiment $F$ using different methods during phantom validation. Top row shows selected frame numbers, and second row shows their corresponding phantom RB images. Other rows display virtual bronchoscopic images generated from tracking results using the methods of Deguchi et al. [4, Schwarz et al. 1], and our new method. Our proposed method shows the best performance.

However, in our experiments, the proposed method still misaligns some RB and VB frames when continuously tracking bronchoscope motions during navigated bronchoscopy. One main reason behind these misalignments might be the shortage of the bending angle information of the bronchoscope bendable part. We did not realize the function of automatically measuring the bending angle. We currently only provide information on the insertion depth and the rotation of the running direction for motion prediction. Otherwise, the nonlinear illumination changes result in dark video images; hence, they cause difficulties of sample weight calculation, and incorrect sample weights unavoidably happen.

Moreover, the following issues must be clarified. First, although our method provides significant tracking accuracy, we clarified that the position and orientation errors shown in Table 1 are stated relative to GTD. However, GTD itself involves errors originating from our manual registration or synchronization. 
Next, since bronchoscopic images were collected inside a static phantom without respiration motion or other deformations, another particular challenge of airway deformation was not explicitly validated in this work. We need to further evaluate our tracking prototype on patient data. Additionally, the computational efficiency of the proposed method is about 1.1 seconds per frame without speed optimizations and multi-threading. Last, although we concentrated on developing bronchoscope motion tracking for navigated bronchoscopy, our prototype should also be appropriate to navigate other endoscopes, e.g., colonoscope.

\section{Conclusions and Future Work}

This paper proposed a novel bronchoscope tracking prototype and its validation on phantom datasets. We realized two functions of our model and applied them to estimate bronchoscope movements. The experimental results demonstrated the accuracy and robustness of our method by pose errors of only $1.10 \mathrm{~mm}$ and $3.88^{\circ}$, successfully registered a total of $7142(86.0 \%)$ images, and increased the tracking performance by at least $23.4 \%$, compared to image- and EMT-based methods. Besides evaluation of our method on patient data and reducing computational times, future work also includes the extension of another function of our bronchoscope prototype and further improvement of its performance.

Acknowledgments. This work was supported in part by Grant-in-Aid for Science Research funded by MEXT and JSPS, and "Chi-no-kyoten" by Aichi Prefecture. Authors would like to thank Dr. H. Tahabatake for providing datasets.

\section{References}

1. Schwarz, Y., Greif, J., Becker, H.D., Ernst, A., Mehta, A.: Real-time electromagnetic navigation bronchoscopy to peripheral lung lesions using overlaid CT images: the first human study. Chest 129(4), 988-994 (2006)

2. Soper, T.D., Haynor, D.R., Glenny, R.W., Seibel, E.J.: In vivo validation of a hybrid tracking system for navigation of an ultrathin bronchoscope within peripheral airways. IEEE TBME 57(3), 736-745 (2010)

3. Deligianni, F., Chung, A.J., Yang, G.Z.: Nonrigid 2-D/3-D registration for patient specific bronchoscopy simulation with statistical shape modeling: Phantom validation. IEEE TMI 25(11), 1462-1471 (2006)

4. Deguchi, D., Mori, K., Feuerstein, M., Kitasaka, T., Maurer Jr., C.R., Suenaga, Y., Takabatake, H., Mori, M., Natori, H.: Selective image similarity measure for bronchoscope tracking based on image registration. MedIA 13(4), 621-633 (2009)

5. Luo, X., Feuerstein, M., Kitasaka, T., Mori, K.: A novel bronchoscope tracking method for bronchoscopic navigation using a low cost optical mouse sensor. In: Wong, K.H., Holmes, D.R. (eds.) SPIE Medical Imaging 2011, Florida USA, vol. 7964 , pp. $79641 \mathrm{~T}(2011)$

6. Isard, M., Blake, A.: Condensation - conditional density propagation for visual tracking. International Journal of Computer Vision 29(1), 5-28 (1998) 
7. Luo, X., Reichl, T., Feuerstein, M., Kitasaka, T., Mori, K.: Modified hybrid bronchoscope tracking based on sequential monte carlo sampler: Dynamic phantom validation. In: Kimmel, R., Klette, R., Sugimoto, A. (eds.) ACCV 2010, Part III. LNCS, vol. 6494, pp. 409-421. Springer, Heidelberg (2011)

8. Arulampalam, M., Maskell, S., Gordon, N., Clapp, T.: A tutorial on particle filters for nonlinear/non-gaussian Bayesian tracking. IEEE TSP 50(2), 174-188 (2002)

9. Schneider, M., Stevens, C.: Development and testing of a new magnetic-tracking device for image guidance. In: Cleary, K.R., Miga, M.I. (eds.) SPIE Medical Imaging 2007, California USA, vol. 6509, pp. 65090I (2007) 